\title{
Applying Bayesian Neural Networks to Event Reconstruction in Reactor Neutrino Experiments
}

\author{
Ye Xu, Weiwei Xu, Yixiong Meng, Kaien Zhu, Wei Xu
}

\author{
Department of Physics, Nankai University, Tianjin 300071, People's Republic \\ of China
}

\begin{abstract}
A toy detector has been designed to simulate central detectors in reactor neutrino experiments in the paper. The electron samples from the Monte-Carlo simulation of the toy detector have been reconstructed by the method of Bayesian neural networks (BNN) and the standard algorithm, a maximum likelihood method (MLD), respectively. The result of the event reconstruction using BNN has been compared with the one using MLD. Compared to MLD, the uncertainties of the electron vertex are not improved, but the energy resolutions are significantly improved using BNN. And the improvement is more obvious for the high energy electrons than the low energy ones.
\end{abstract}

Keywords: Bayesian neural networks, Event reconstruction, Neutrino oscillation

PACS numbers: 07.05.Mh, 29.85.Fj, 14.60.Pq

\section{Introduction}

The main goals of reactor neutrino experiments are to detect $\overline{\nu_{e}} \rightarrow \overline{\nu_{x}}$ oscillation and precisely measure the mixing angle of neutrino oscillation $\theta_{13}$. The experiment is designed to detect reactor $\overline{\nu_{e}}$ 's via the inverse $\beta$-decay reaction

$$
\overline{\nu_{e}}+p \rightarrow e^{+}+n
$$

The signature is a delayed coincidence between $e^{+}$and the neutron captured signals. It is very important to reconstruct the energy and the vertex of a signal detected in the experiments. The standard algorithm of the event reconstruction in the experiments is a maximum likelihood method (MLD from now on). But the method of Bayesian neural networks (BNN from now on)[1] is more suitable than MLD for the event reconstruction of reactor neutrino experiments. BNN is an algorithm of the neural networks trained by Bayesian statistics. It is not only a non-linear function, but also controls model complexity. So its flexibility makes it possible to discover more general relationships in data than the traditional statistical methods and its preferring simple models make it possible to solve the over-fitting problem better than the general neural networks[2]. In this paper,

\footnotetext{
${ }^{*}$ Corresponding author, e-mail address: xuye76@nankai.edu.cn
} 
BNN is applied to the event reconstruction of the electron samples from the Monte-Carlo simulation of a toy detector of reactor neutrino experiments. And the result of the event reconstruction using BNN is compared with the one using MLD.

\section{The Regression with Bayesian Neural Networks[1, 3]}

The idea of BNN is to regard the process of training a neural network as a Bayesian inference. Bayes' theorem is used to assign a posterior density to each point, $\bar{\theta}$, in the parameter space of the neural networks. Each point $\bar{\theta}$ denotes a neural network. In the method of BNN, one performs a weighted average over all points in the parameter space of the neural network, that is, all neural networks. The methods make use of training data $\left\{\left(x_{1}, t_{1}\right),\left(x_{2}, t_{2}\right), \ldots,\left(x_{n}, t_{n}\right)\right\}$, where $t_{i}$ is the known target value associated with data $x_{i}$, which has $P$ components if there are $P$ input values in the regression. That is the set of data $x=\left(x_{1}, x_{2}, \ldots, x_{n}\right)$ which corresponds to the set of target $t=\left(t_{1}, t_{2}, \ldots, t_{n}\right)$. The posterior density assigned to the point $\bar{\theta}$, that is, to a neural network, is given by Bayes' theorem

$$
p(\bar{\theta} \mid x, t)=\frac{p(x, t \mid \bar{\theta}) p(\bar{\theta})}{p(x, t)}=\frac{p(t \mid x, \bar{\theta}) p(x \mid \bar{\theta}) p(\bar{\theta})}{p(t \mid x) p(x)}=\frac{p(t \mid x, \bar{\theta}) p(\bar{\theta})}{p(t \mid x)}
$$

where data $x$ do not depend on $\bar{\theta}$, so $p(x \mid \theta)=p(x)$. We need the likelihood $p(t \mid x, \bar{\theta})$ and the prior density $p(\bar{\theta})$, in order to assign the posterior density $p(\bar{\theta} \mid x, t)$ to a neural network defined by the point $\bar{\theta} \cdot p(t \mid x)$ is called evidence and plays the role of a normalizing constant, so we ignore the evidence. That is,

$$
\text { Posterior } \propto \text { Likelihood } \times \text { Prior }
$$

We consider a class of neural networks defined by the function

$$
y(x, \bar{\theta})=b+\sum_{j=1}^{H} v_{j} \sin \left(a_{j}+\sum_{i=1}^{P} u_{i j} x_{i}\right)
$$

The neural networks have $P$ inputs, a single hidden layer of $H$ hidden nodes and one output. In the particular BNN described here, each neural network has the same structure. The parameter $u_{i j}$ and $v_{j}$ are called the weights and $a_{j}$ and $b$ are called the biases. Both sets of parameters are generally referred to collectively as the weights of the BNN, $\bar{\theta} . y(x, \bar{\theta})$ is the predicted target value. We assume that the noise on target values can be modeled by the Gaussian distribution. So the likelihood of $n$ training events is

$$
p(t \mid x, \bar{\theta})=\prod_{i=1}^{n} \exp \left[-\left(\left(t_{i}-y\left(x_{i}, \bar{\theta}\right)\right)^{2} / 2 \sigma^{2}\right]=\exp \left[-\sum_{i=1}^{n}\left(t_{i}-y\left(x_{i}, \bar{\theta}\right) / 2 \sigma^{2}\right)\right]\right.
$$


where $t_{i}$ is the target value, and $\sigma$ is the standard deviation of the noise. It has been assumed that the events are independent with each other. Then, the likelihood of the predicted target value is computed by Eq. (4).

We get the likelihood, meanwhile we need the prior to compute the posterior density. But the choice of prior is not obvious. However, experience suggests a reasonable class is the priors of Gaussian class centered at zero, which prefers smaller rather than larger weights, because smaller weights yield smoother fits to data. In the paper, a Gaussian prior is specified for each weight using the Bayesian neural networks package of Radford Neal ${ }^{1}$. However, the variance for weights belonging to a given group(either input-to-hidden weights $\left(u_{i j}\right)$, hidden -biases $\left(a_{j}\right)$, hidden-to-output weights $\left(v_{j}\right)$ or output-biases $\left.(b)\right)$ is chosen to be the same: $\sigma_{u}^{2}, \sigma_{a}^{2}, \sigma_{v}^{2}, \sigma_{b}^{2}$, respectively. However, since we don't know, a priori, what these variances should be, their values are allowed to vary over a large range, while favoring small variances. This is done by assigning each variance a gamma prior

$$
p(z)=\left(\frac{\alpha}{\mu}\right)^{\alpha} \frac{z^{\alpha-1} e^{-z \frac{\alpha}{\mu}}}{\Gamma(\alpha)}
$$

where $z=\sigma^{-2}$, and with the mean $\mu$ and shape parameter $\alpha$ set to some fixed plausible values. The gamma prior is referred to as a hyperprior and the parameter of the hyperprior is called a hyperparameter.

Then, the posterior density, $p(\bar{\theta} \mid x, t)$, is gotten according to Eqs. (2),(4) and the prior of Gaussian distribution. Given an event with data $x^{\prime}$, an estimate of the target value is given by the weighted average

$$
\bar{y}\left(x^{\prime} \mid x, t\right)=\int y\left(x^{\prime}, \bar{\theta}\right) p(\bar{\theta} \mid x, t) d \bar{\theta}
$$

Currently, the only way to perform the high dimensional integral in Eq. (6) is to sample the density $p(\bar{\theta} \mid x, t)$ with the Markov Chain Monte Carlo (MCMC) method $[1,4,5,6]$. In the MCMC method, one steps through the $\bar{\theta}$ parameter space in such a way that points are visited with a probability proportional to the posterior density, $p(\bar{\theta} \mid x, t)$. Points where $p(\bar{\theta} \mid x, t)$ is large will be visited more often than points where $p(\bar{\theta} \mid x, t)$ is small.

Eq. (6) approximates the integral using the average

$$
\bar{y}\left(x^{\prime} \mid x, t\right) \approx \frac{1}{L} \sum_{i=1}^{L} y\left(x^{\prime}, \bar{\theta}_{i}\right)
$$

where $L$ is the number of points $\bar{\theta}$ sampled from $p(\bar{\theta} \mid x, t)$. Each point $\bar{\theta}$ corresponds to a different neural network with the same structure. So the average is an average over neural networks, and is closer to the real value of $\bar{y}\left(x^{\prime} \mid x, t\right)$, when $L$ is sufficiently large.

\footnotetext{
${ }^{1}$ R. M. Neal, Software for Flexible Bayesian Modeling and Markov Chain Sampling, http://www.cs.utoronto.ca/ radford/fbm.software.html
} 


\section{Toy Detector and Simulation}

\subsection{Toy Detector}

In the paper, a toy detector is designed to simulate central detectors in the reactor neutrino experiments, such as Daya Bay experiment[7] and Double Chooz experiment[8], with CERN GEANT4 package[9]. The toy detector consists of three regions, and they are the Gd-doped liquid scintllator(Gd-LS from now on), the normal liquid scintillator(LS from now on) and the oil buffer, respectively. The toy detector of cylindrical shape like the detector modules of Daya Bay experiment and Double Chooz experiment is designed in the paper. The diameter of the Gd-LS region is 2.4 meter, and its height is 2.6 meter. The thickness of the LS region is 0.35 meter, and the thickness of the oil part is 0.40 meter. In the paper, the Gd-LS and LS are the same as the scintillator adopted by the proposal of the CHOOZ experiment[10]. The 8-inch photomultiplier tubes (PMT from now on) are mounted on the inside the oil region of the detector. A total of 366 PMTs are arranged in 8 rings of 30 PMTs on the lateral surface of the oil region, and in 5 rings of $24,18,12,6,3$ PMTs on the top and bottom caps.

\subsection{Monte-Carlo Simulation of Toy Detector}

The response of the electron events deposited in the toy detector is simulated with GEANT4. Although the physical properties of the scintillator and the oil (their optical attenuation length, refractive index and so on) are wave-length dependent, only averages[10] (such as the optical attenuation length of Gd-LS with a uniform value is 8 meter and the one of LS is 20 meter) are used in the detector simulation. The program couldn't simulate the real detector response, but this won't affect the result of the comparison between BNN and MLD. The program allows us to simulate the detector response for the electron events of the different energy and vertex. In the paper, 10000 electron events regarded as the training sample are uniformly generated throughout Gd-LS region and their energy is also uniformly generated from $1 \mathrm{MeV}$ to $13 \mathrm{MeV}$. 3000 electron events regarded as the $1 \mathrm{MeV}$ test sample are generated uniformly throughout Gd-LS region. The test samples from $2 \mathrm{MeV}$ to $8 \mathrm{MeV}$ are generated in the same way, respectively.

\section{Event Reconstruction}

The task of the event reconstruction in the reactor neutrino experiments is to reconstruct the energy and the vertex of a signal. The maximum likelihood method is a standard algorithm of the event reconstruction in the reactor neutrino experiments. The likelihood is defined as the joint Poisson probability of observing a measured distribution of photoelectrons over the all PMTs for given $(E, \vec{x})$ coordinates in the detector. The Ref.[11] for the work of the CHOOZ experiment shows the method of the reconstruction in detail. The algorithm of BNN is also applied to event reconstruction, and its result is compare with the one of MLD.

\subsection{Event Reconstruction with MLD}

In the paper, the event reconstruction with the MLD are performed in the similar way with the CHOOZ experiment[11], but the detector is different from the detec- 
tor of the CHOOZ experiment, so compared to Ref.[11], there are some different points in the paper:

(1) The detector in the paper consists of three regions, so the path length from a signal vertex to the PMTs consist of three parts, and they are the path length in Gd-LS region, the one in LS region, and the one in oil region, respectively.

(2) Considered that not all PMTs in the detector can receive photoelectrons when a electron is deposited in the detector, the $\chi^{2}$ equation is modified in the paper and different from the one in the CHOOZ experiment, that is, $\chi^{2}=$ $\sum_{N_{j}=0} \bar{N}_{j}+\sum_{N_{j} \neq 0}\left(\bar{N}_{j}-N_{j}+N_{j} \log \left(\frac{N_{j}}{N_{j}}\right)\right)$, where $N_{j}$ is the number of photoelectrons received by the j-th PMT and $\bar{N}_{j}$ is the expected one for the j-th PMT[11].

(3) $c_{E} \times N_{\text {total }}$ and the coordinates of the charge center of gravity for the all visible photoelectrons from a signal are regarded as the starting values for the fit parameters $(E, \vec{x})$, where $N_{\text {total }}$ is the total numbers of the visible photoelectrons from a signal and $c_{E}$ is the proportionality constant of the energy $E$, that is, $E=c_{E} \times N_{\text {total }}$. $c_{E}$ is obtained through fitting $N_{\text {total }}$ 's of the $1 \mathrm{MeV}$ electron events, and is $\frac{1}{235 / \mathrm{MeV}}$ in the paper.

$(E, \vec{x})$ of the all electron events, including the test sample and the training sample, are reconstructed using MLD.

\subsection{Event Reconstruction with BNN}

In the paper, the Cartesian coordinates $(x, y, z)$ of the all events, including the test sample and the training sample, are transformed to their cylindrical coordinates $(r, \theta, z)$. The $(E, r, \theta, z)$ are used as inputs to the BNN, which have the input layer of 4 inputs, the single hidden layer of 8 nodes and the output layer of a output which is $E$, or $x, y, z$, respectively. The $E$ and $x, y, z$ of the test samples are predicted using the BNN, respectively. A Markov chain of neural networks is generated using the Bayesian neural networks package of Radford Neal, with the training sample, in the process of the event reconstruction. One thousand iterations, of twenty MCMC steps each, are used in the paper. The neural network parameters are stored after each iteration, since the correlation between adjacent steps is very high. That is, the points in neural network parameter space are saved to lessen the correlation after twenty steps. It is also necessary to discard the initial part of the Markov chain because the correlation between the initial point of the chain and the points of the part is very high. The initial three hundred iterations are discarded in the paper.

\section{Conclusion}

Fig.1, Fig.2 and Fig.3 illustrate the results of the event reconstruction with BNN and MLD. Fig. 1 shows that the errors of the vertex of $1 \mathrm{MeV}$ and $8 \mathrm{MeV}$ electrons reconstructed by the BNN are consistent with the ones by MLD, that is, they are not obviously different. Fig. 2 shows that the energy uncertainty for 1 $\mathrm{MeV}$ electrons with BNN decreases by $95.0 \%$ in comparison with the one using MLD. And the uncertainty in the case of the $8 \mathrm{MeV}$ events decreases by $76.3 \%$. Fig. 3 shows the energy resolutions using BNN are more significantly improved in comparison with the one using MLD while increasing energy. Meanwhile, the relative errors of the energy resolutions are about $2.0 \%$, and are from fit errors (about 1.5\%) and statistical errors (about 1.3\%). So the difference between 
results of BNN and MLD is not significant in the case of $1 \mathrm{MeV}$ events in consideration of the effect of statistical fluctuations. But the contribution to the difference is mainly from the superiority of BNN for the events from $2 \mathrm{MeV}$ to 8 $\mathrm{MeV}$. Thus it can be seen that the energy resolutions using BNN are significantly improved for the high energy events in comparison with the one using MLD. Therefore, BNN can be well applied to the energy reconstruction in the reactor neutrino experiments, and the better energy resolution is obtained by BNN.

Although the discussion in the paper are only for the reactor neutrino experiments, it is expected that the algorithm of BNN can also be applied to the event reconstruction of the other experiments and will find wide application in the experiments of high energy physics.

\section{Acknowledgements}

This work is supported by the National Natural Science Foundation of China(NSFC) under the contract No. 10605014.

\section{References}

[1] R. M. Neal, Bayesian Learning of Neural Networks, 1st edn. (Springer-Verlag, New York, 1996)

[2] R. Beale and T. Jackson, Neural Computing: An Introduction, 1st edn. (Adam Hilger, New York, 1991)

[3] P. C. Bhat and H. B. Prosper, in Proceedings of Statistical Problems in Particle Physics, Astrophysics and Cosmology, Oxford, UK 12-15, September 2005, edited by L. Lyons and M. K. Unel, p. 151

[4] S. Duane, A. D. Kennedy, B. J. Pendleton and D. Roweth, Physics Letters B 195, 216 (1987)

[5] M. Creutz and A. Gocksch, Physical Review Letters 63, 9 (1989)

[6] P. B. Mackenzie, Physics Letters B 226, 369 (1989)

[7] Daya Bay Collaboration, Daya Bay Proposal: A Precision Measurement of the Neutrino Mixing Angle $\theta_{13}$ Using Reactor Antineutrino At Daya Bay, arXiv: hep-ex/0701029

[8] F. Ardellier et al., Double Chooz: A Search for the Neutrino Mixing Angle $\theta_{13}$, arXiv: hep-ex/0606025

[9] Geant4 Reference Manual, vers. 9.0 (2007)

[10] The CHOOZ Experiment Proposal (1993), available at the WWW site http://duphy4.physics.drexel.edu/chooz_pub/

[11] M. Apollonio et al., European Physical Journal C 27, 331 (2003) 


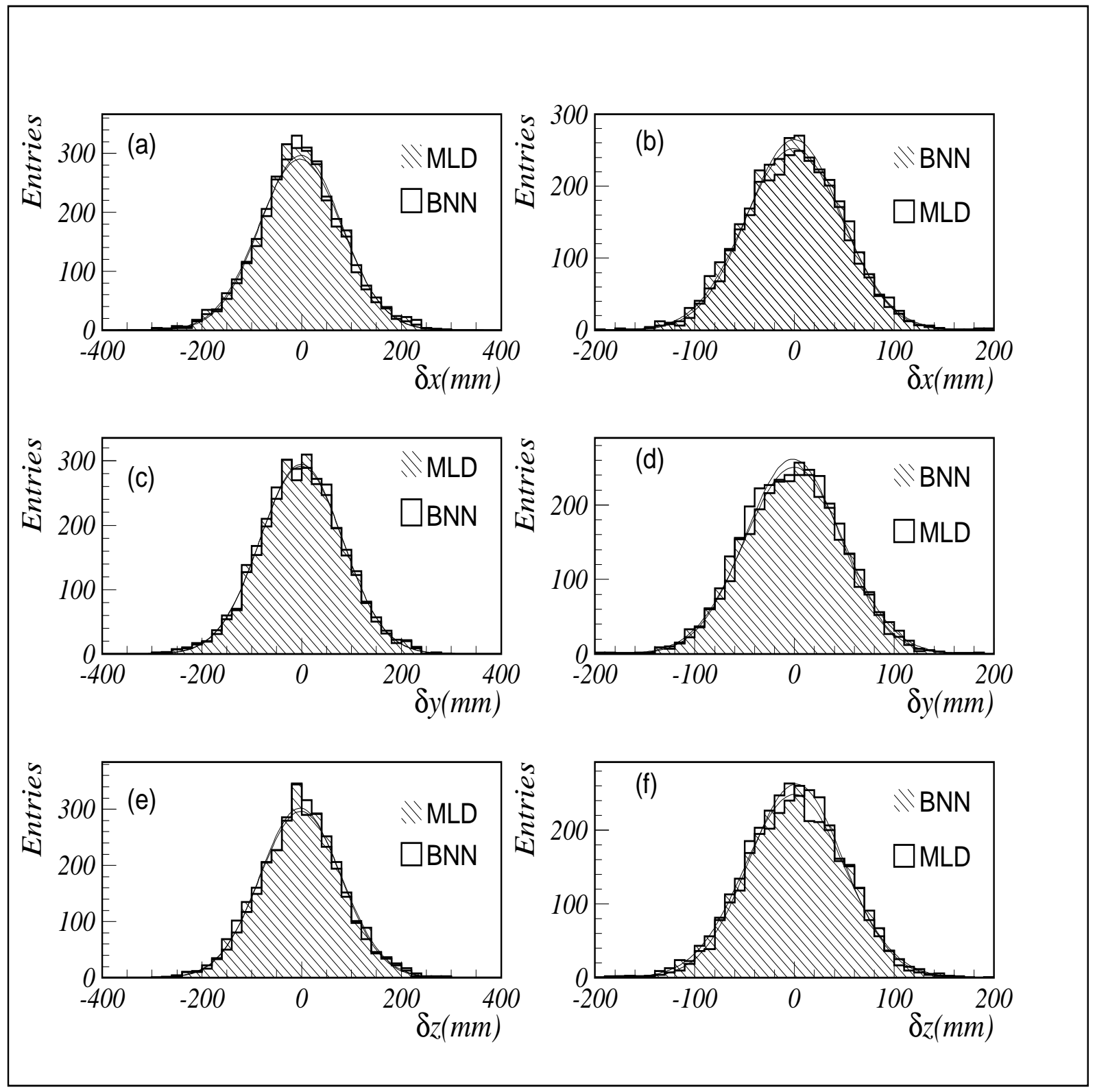

Fig. 1: $\delta x, \delta y, \delta z$ is the difference between the coordinates of the reconstructed position and the generated ones, respectively. The event position is reconstruted using BNN and MLD, respectively. (a)(c)(e) illustrate the difference distribution of the $1 \mathrm{MeV}$ electrons, and (b)(d)(f) illustrate the one of 8 $\mathrm{MeV}$ electrons. 


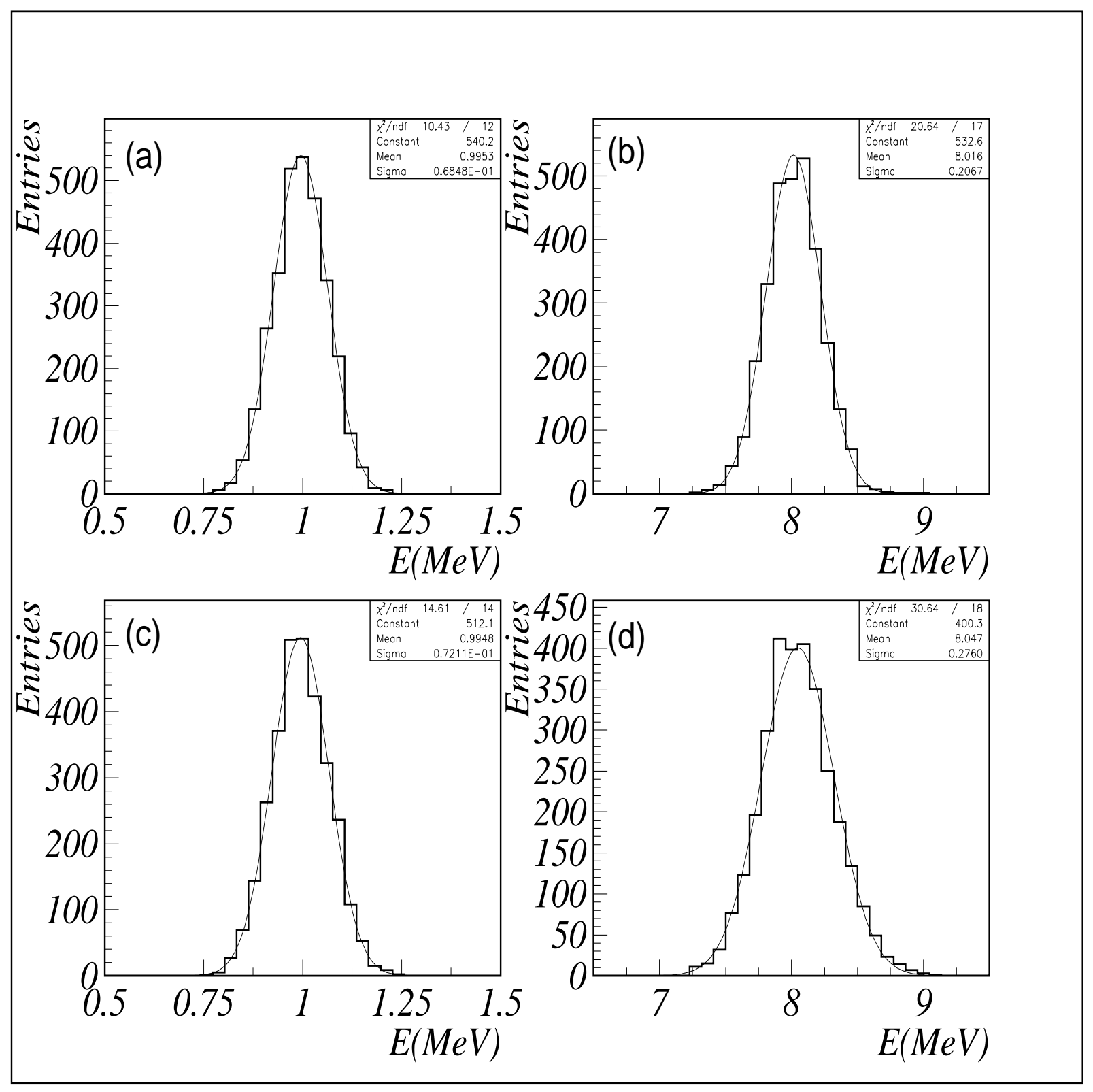

Fig. 2: The energies of $1 \mathrm{MeV}$ and $8 \mathrm{MeV}$ electrons are reconstructed using BNN and MLD, respectively. (a), (b) illustrate the distribution of the energy reconstructed by $\mathrm{BNN}$ for $1 \mathrm{MeV}$ and $8 \mathrm{MeV}$ electrons, respectively. (c), (d) illustrate the distribution of the energy reconstructed by MLD for 1 $\mathrm{MeV}$ and $8 \mathrm{MeV}$ electrons, respectively. 


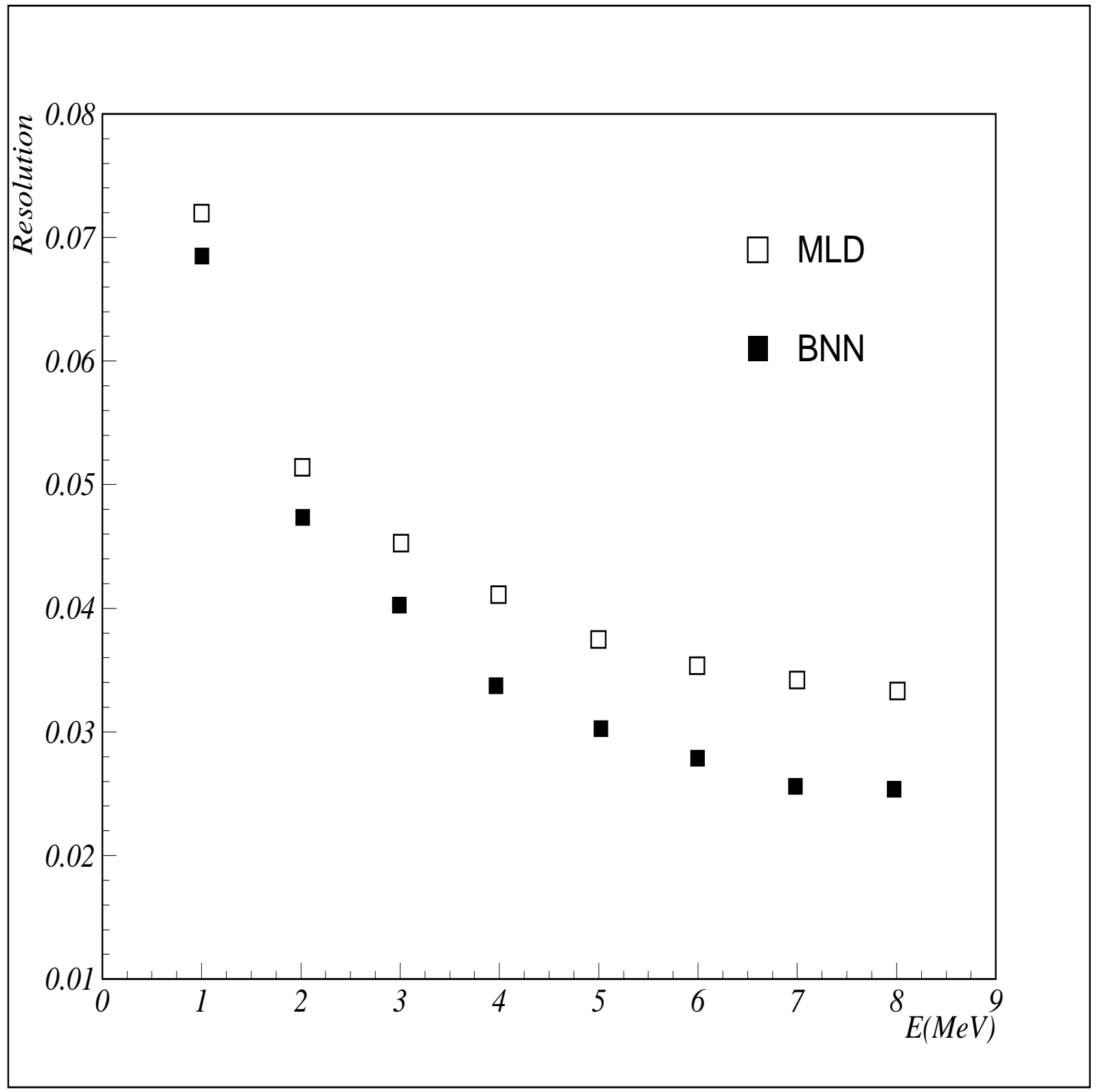

Fig. 3: The energy resolution of the test sample from $1 \mathrm{MeV}$ to $8 \mathrm{MeV}$ are shown in the figure. The white squares denote the resolutions using MLD, and the black squares denote the one using BNN. 